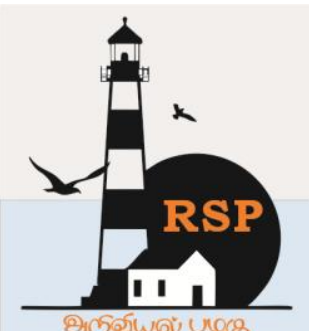

INTERNATIONAL RESEARCH JOURNAL ON ADVANCED SCIENCE HUB

RSP SCIENCE HUB

(The Hub of Research Ideas)

Available online at www.rspsciencehub.com

Special Issue of First International Conference on Engineering, Science, and Technology (ICEST 2021)

\title{
Cloud Technology and Return on Investment (ROI)
}

Vikas R Gangadhar ${ }^{1}$, Dr. Ajim Shaikh ${ }^{2}$

${ }^{1}$ Research Scholar, MIT Art, Design and Technology University, Pune, Maharashtra, India

${ }^{2}$ Assistant Professor \& Guide, MIT Art, Design and Technology University, Pune, Maharashtra, India.

vikasgangadhar2012@gmail.com ${ }^{1}$

\begin{abstract}
Many organizations across the globe have adopted cloud technology or they are in process of migrating their existing IT setup to cloud platform. Are they really reaping the benefits of cloud technology? The cloud service providers play a vital role by providing technology inside and right direction to their clients. Companies will not opt for cloud technology unless they see the real benefits to their business. They will invest into the proposal, which will provide them higher returns. Adopting cloud technology certainly provides multiple levers for improving the returns on investment (ROI). One should choose a right cloud solution by following best practices and adopting the right cloud strategy for their own business solution. ROI is most widely used for financial success in trades. ROI is the equivalent increase in the value on your investment over a period of time. The basic ways to improve ROI are to reduce the investment, increase revenue, decrease operational costs, and start the early returns.Cloud computing should not be seen as a technical infrastructure advancement then we will be missing the wider picture of the impact of technology over the business outcomes. The value proposition matters most, and it does not always represent the financial performance. It can be customer value, corporate value or market brand value. Those aspects should not be ignored.This paper covers the drivers, which contributes to ROI and shows how to use these levers to get maximum benefits.
\end{abstract}

Keywords: Cloud Technology, Return on Investment, Cloud Migration

\section{Introduction}

By now, most of the organizations are aware about the cloud offerings and benefits out of cloud adoption. This is the major reasons why organizations are moving towards cloud adoption, but sometimes blind move can worsen the situation. Many organizations assume that shifting workload from in-house infrastructure to the cloud platform is a simple process and will being lots of efficiency with less pay. It is not as simple as it looks without well thought cloud strategy. The best way to avoid the common mistakes and pitfalls is to know all about the cloud platform and follow best practices. These tricks of trade will help you to earn a bigger ROI against cloud deployment investment.Cloud computing is not entirely new concept, but it is combination of new and existing technologies. Following are the key promises brought by cloud computing primarily due to its technical characteristics:

- The ability to provision infinite system resources and consistence performance for smaller number of users to few thousand users maintaining same service-levels.

- Serverless computing which is not locked to specific infrastructure components or locations.

- Pay-as-you-go usages with minimal initial investment. Metered connection usages and ownership lies with service provider. 
- Elasticity of Service and able to scale up and scale down instantly. No provisioning delays and advance forecast is required.

- Anywhere and anytime access feature provides near $100 \%$ uptime commitment.

These technical features are found in many other technology solutions. The differential promises of cloud technology are at the rate of change, scale of cost reduction and specific system performance much higher over traditional technologies. The differentiator of cloud technology is not just the infrastructure utility services but it includes all higher-level offerings that enhance business values. It has been seen the trend of movement from IT centric services to business centric services across information technology spectrum. Cloud offers business centric application with business process delivery as it is in core support. Additionally, assured security measures cover essential services where clients are always ready to pay extra for these values proposition.Cloud technology is making impact over revenue and budgeting. Virtualization, economies of scale and lower up-front investment improves cost v/s performance ratio. The investment needs to be thoughtfully balanced against cloud offerings and its value-added services.

\section{ROI Consideration}

There are various cost elements that can influence overall ROI. These include the initial up-front investment, implementation cost and operational cost.

\subsection{Initial up-front investment:}

Cloud platform need initial capital expenses (capex) that includes defining and designing architecture, establishing security standards, setting up governance process, training and hiring cloud experts etc.

\subsection{Implementation cost:}

The implementation cost include the cost of application migration, modernization, managing parallel environments during project execution, hardware costs etc. It is important to carry out the proper assessment. This will avoid unwanted and wrong investments. The proper due diligence required in selecting right cloud provider, landing zone, sizing and right approach on integration and consolidation of system components. Licensing cost may have large impact while choosing OS/DB platform during migration. This will influence migration strategy and in turn implementation approach and timelines.

\subsection{Operational cost:}

Up keeping and high availability of application hosted on cloud platform is responsibility of cloud supplier. The service level agreement drives the operational cost. The cloud platform brings new tools for infrastructure monitoring and governance. There will be additional cost of running on-premise system before DC exit. There will be hiring cost associated with talent acquisition. Cost can be further reduced by using right size VMs, referring to recommendations and offers extended by cloud supplier and by consolidating storage requirements.Measuring benefits or success of the cloud adoption is not a direct measure of cost. It involves multiple $y$ direct and indirect costs. Success is not directly linked to IT cost as business values and financial outcomes play major roles. Hence it all links goals and achievement of the organization. ROI should comprise clear definition at the beginning to measure it upon completion.

\section{Cloud Migration Strategy}

Cloud migration requires comprehensive strategy through planning and setting up the migration goals, timeline, reviewing the anticipates challenges and risks for successful project delivery.Migration strategies should consider the workloads planned for migration to the cloud, which will remain on-premise, and the new capabilities on-boarded as part of cloud journey. Best way to deal with planning process to refer to use cases and cloud migration best practices. The migration approach demands different treatments for different mission-critical applications, backup and recovery, in-flight projects, productivity and collaboration tools. A solid strategy will set up the foundation for seamless process for successful migration.Migration strategies should cover the risk assessments exercise, budgeting process and security consideration. The type of cloud offering consideration such as public or private that will host specific workloads being migrated. Migration strategies to consider simplified and consistent management approach for cloud platform. The security aspect of data encryption should be considered for data in rest or for data in transit along with regulatory and compliance 
requirements. Migration strategy should comprise a communication plan to all stakeholders. Using exiting knowledge, company can prepare a plan, which can be altered based on further learnings from subsequent phases. The complexity of plan varies on the exiting architecture and its licensing agreements Typically the migration should start with less complex application as quick win and progress towards high complexity application at the end.

Stephen(2011) has advocated the 6 most common application migration strategies in his blog.

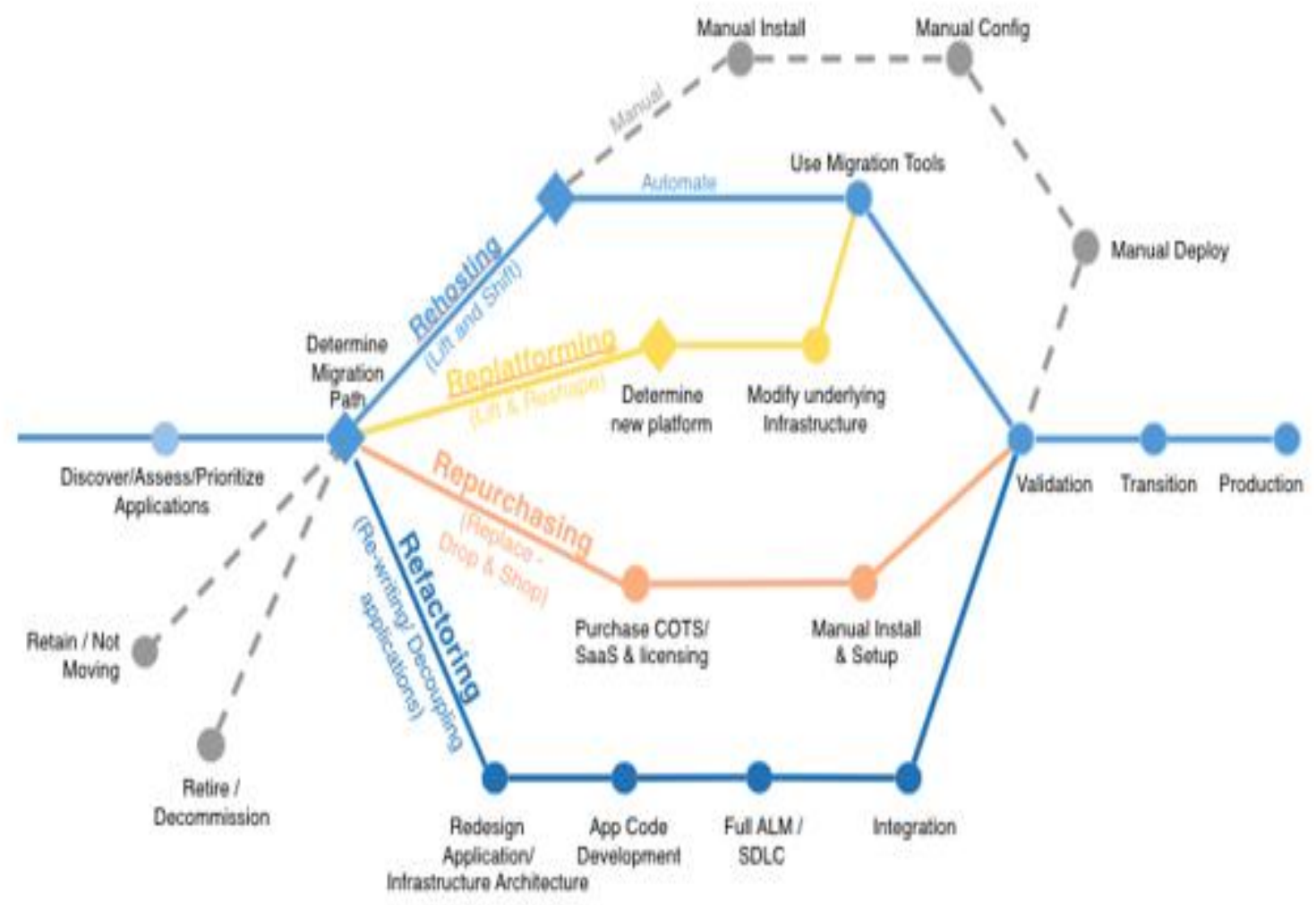

Fig.1. Application Migration strategies,https://aws.amazon.com, n.d.

\section{A) Lift and shift: Rehosting}

The existing application are migrated as-is to cloud platform without considering further optimization. The existing OS/DB platform will remain same. By sampling migrating to cloud platform has benefited client to reduce their IT operational cost. Organization can optimize their application or rearchitect the platform while they are running over cloud services easily. The cloud service provider will assist on application migration, which needs skilled resource and usages of specialized tools.

\section{B) Lift think and shift: Replatforming}

While considering plan for lift and shift option, the quicker optimization possibilities are varied and adopted in migration process. The typical example of OS/DB migration while moving on cloud journey. There are services offered by cloud providers for improved database management such as Database as a Service (DaaS). This brings tangible benefit without changing the application and platform architecture. Company has saved on licensing costs and enhance the supportability of products, which might have gone out of support.

\section{C) NewProductimplementation: Repurchasing}

The organization may consider green field implementation of new product replacing use case of existing product to bring enhancement, more efficiency and agility. Range of SaaS products are preferred to bring real cloud benefits.

\section{D) Refactoring / Re-architecting}

The application is re-imagined using new architecture and development tools using cloudnative features.Mainly this kind of migration has driven through strong business case for additional features, for better performance and scalability. This is not possible with any of the above migration approaches.This need more afront 
investment, but the product will be fit for purpose and will being most beneficial.

\section{E) Decommissioning: Retire}

Post migration, the legacy application needs to be decommissioned as soon as possible to get rid of technical debt. These will save cost and increase the usage of your new application

\section{F) No option/use case for Cloud Migration: Retain}

There will be a still application, which does fit into any of the use case. We cannot get rid of them, as they do not have suitable cloud landing zone and still part of organizational application estate. Or the use case does not provide substantial use for migrating them over cloud platform. Microsoft has emphasised that the cloud adoption needs major organizational change management across people, process, and technology. A holistic approach will lead to success cloud journey and ensure that your organization realizes benefits of cloud adoption such as efficiency, scale and agility once you migrate your workloads to the cloud.

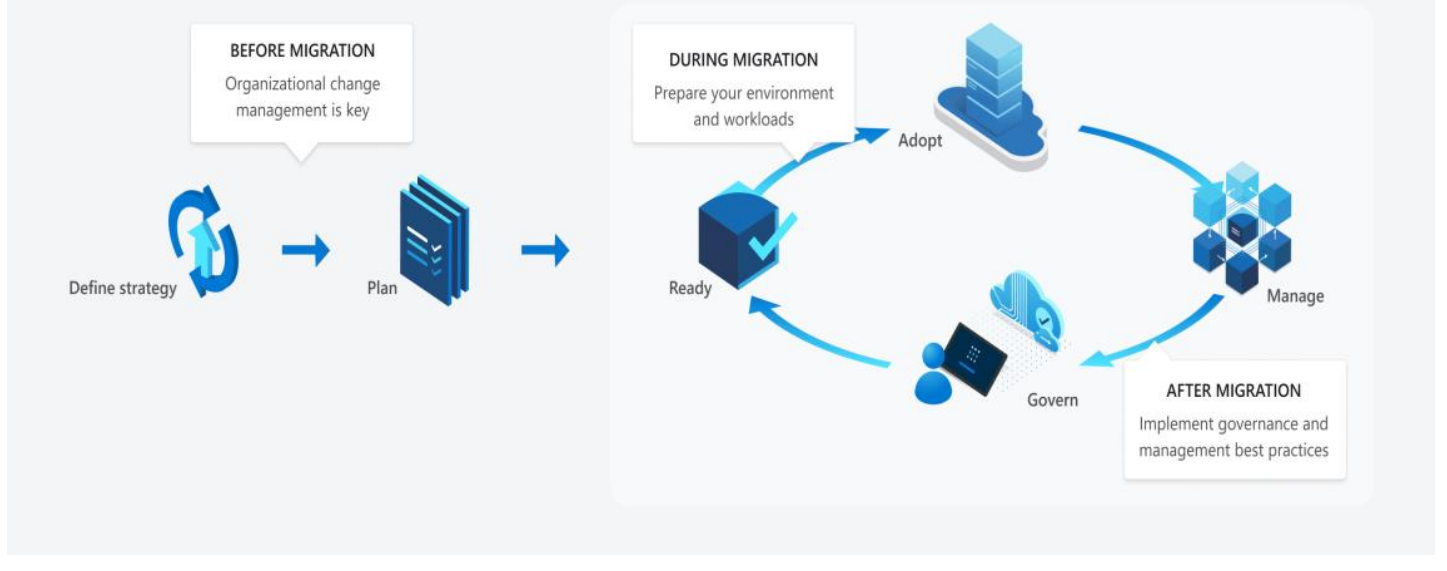

Fig.2. Migration Journey, https://azure.microsoft.com, n.d.

Microsoft suggests key consideration during each stage of your cloud journey.

\subsection{Before Migration}

Define your migration strategy based on business case, business goals and priority of workload to be migrated to new platform. Target the businesscritical workload first to receive continuous funding for migration project. Build the cloud capabilities and engage all stakeholders to secure broad support. Start preparing actual plan by going through your existing inventory and their interdependences. Review sizing guidelines, exiting workload, performance metrics and cost estimates for potential cost saving. Microsoft has provided tools for calculating your total cost of ownership (TCO). The variance between your existing on-premise operation cost and azure TCO will provide you potential benefits against cloud investment.Break up overall plan into smaller milestones and get buying from key stakeholders.

\subsection{During Migration}

Groom local IT team and business teams into cloud technology. Let them learn and understand the migration tools and new operational process. Prepare the cloud infrastructure as landing zone for migration activities.

Azure provides pre-configured landing zone for networking, for security and identity services and for management and governance. Follow best practices by balancing agility and your company's customized standards to avoid post migration governance issues.

Perform the actual migration steps based on your horizontal or vertical application migration approach. Diligently review the options and tool provided by Azure for workload migration. The mocks and dry runs will provide you sufficient leads in overall migration cutover for production operation. Keeping on re-visiting your plan based on learning from each iteration.

\subsection{After Migration}

As a first thing, get the fill control and visibility on security posture. Use Azure native tools to detect and respond to threads quickly. Review the workload and recommendation over compliance and cost saving for utilizing the cloud services optimally. Ensure that your workload is protected through backup and disaster recovery solutions. Monitor and adjust the cloud spending for optimal ROI. 
Cloud technology provides us a huge opportunity to provide substantial returns on investment, but only when you execute the strategy well against ROI killers.

To achieve higher savings, an organization must execute its cloud strategy quickly and efficiently. The longer execution time of migration for legacy applications will increase expenses and disruption in services. The IT operation cost goes up due to dual platform maintenance.

\section{Risk and Threats}

ROI calculation is the most challenging part of cloud migration. As a human being, we have a tendency to undervalue the expenses by ignoring certain heads in the entire migration process. We focus only on visible cost but there are many items such as usages of tool and services utilized during transition process. The network setup cost involved for handling increased demand during migration and post migration operational cost. Though the cloud migrations will provide cost benefits, but one should consider installation cost of new technology and its ongoing operational cost while budgeting and planning.

Financial planning is must for successful cloud migration. While setting up a budget, the cost associated with the existing applications, cost of moving these applications and cost of running these applications over cloud platform need to be considered.

Many companies have hired external consulting firm for accurate budget planning and such firm work closely with internal teams, cloud service providers and financial experts for conducting the necessary assessments and prepare the accurate budget projection.

Similar to any other IT projects, cloud migration project also carries some risks. The proper risk assessment and mitigation planning will avoid the delays, unexpected roadblocks and trend towards project success.

The risks and threats will arise from two major groups.

1. Governance related

2. Technical related

\subsection{Governance related}

- Selecting the right team

Cloud migration need involvement of crossfunctional teams and external vendors. Identifying the key team members from varied skills and ensuring that all of them come to common concise by avoiding conflicting situation is key challenge. Needed a cloud advocator with strong negotiation skills.

\section{- Unrealistic goal setting}

The cultural barricades may slow down the cloud initiatives. For many companies, cloud computing is major shift in mind set of people. Sometimes over selling of cloud, benefits may raise an unrealistic expectation about cloud platform.

Shifting to the cloud technology requires major transformations. Companies should set up realistic goals by setting up smaller multiple milestones. Let us start with easy options and quick wins to showcase the cloud benefits to boost the confidence of team.

\section{- Poor project execution}

Project orchestration is key for success. The entire use case of cloud computing is based on cost saving and optimization of resources. The poor planning will delay the project execution timelines resulting into extra spending. Sponsors may stop funding if they do not see the benefits.

Project management team should be experienced in handling cloud migration projects. It is recommended to target a small workload for initial migration and let an organization iterate through setting up necessary security controls and operational governance.

Project management to ensure that the project team are trained and understand the key cloud concepts. The cloud adoption needs agile methodology over traditional waterfall delivery model. The project management should be seasoned to handle difficult situation and bring back the project on track from turmoil.

Diligent planning and close tracking of project deliverables will ensure project success.

\section{- Mishandling of change management}

Cloud computing has brought radical change in how the applications are used, build and managed. The DevOps has brought an agility in the way we deliver the traditional changes and bug fixes during software development lifecycle.

\subsection{Technical related}

- Architectural complexity:

When organization move workloads towards cloud platform, normally they think about usages of public and private clouds for migrating onpremise environments as a safer approach. This 
hybrid environment may quickly grow into complex architecture, which will be difficult to manage. The misaligned in compatibility brings inconsistency and interoperability problems while handling workload across platform. This needs a proper planning and vision while designing cloud architecture.

\section{- Poor application selection:}

It is vital to avoid choosing applications to be migrated to the cloud that are well suited to operate on-premise landscape. This selection of workloads depends on your organization usages and business needs. Factors, which influence the decision-making, are network latency, security and controls, volumes of data processed to and forth between cloud and on-premise landscape.

\section{- Application dependencies:}

Some of the applications are tightly integrated and have specific latency requirement. We should consider the relationship among various system components such as application servers, databases and other services for smooth operation. Perform the proper due diligence and avoid a situation in which some part of integrated system has left behind by mistake.

\section{- Unwanted latency:}

Latency issues can occur while accessing application over cloud platform. Some applications are very sensitive about latency issues. To prevent latency issues, consider keeping these applications on-premise platform or ensure that cloud supplier has provisioned necessary network bandwidth availability to avoid malfunctioning of services.

\section{- Security considerations:}

Migrating your organization data to public cloud platform poses security risks. To address this concern related data security and privacy, choose vendor who has consistent track record of providing secured network connections. The cloud supplier should have the necessary tools, practices and policies to conform with appropriate security requirements.

\section{Conclusions}

Well thought cloud deployment strategy would yield maximum returns over investments. The results can be pursed as monetary benefits in terms of profit or non- monetary benefits in terms if increased business value, employee productivity and satisfaction. Cloud migration is something more than lift-and-shift. Our deliberate effort for application modernization for improvement of efficiency will realize into more cost savings.Many organizations have adopted emerging trends of cost reduction by leveraging cloud platform. The leading IT suppliers are address customer needs by vigorously evaluated various option in optimizing the hardware, software and services over cloud platform. The difficulties and challenges will be there in the cloud migration process of moving onpremise legacy applications to newer capabilities. They must be articulated in a language that business team can understand and relate to cost investment, improvements and business performance. The IT team to leverage their products and services for defending attack and support customers and increase their market shares. It is recommended follow well thought customized approach to get on the cloud as quickly as possible with smaller workload at a time. Let us focus on smaller and manageable millstones that showcase business value at early stages while your IT staff learning and improvising their cloud experience and expertise. There are wide range of applications can be hosted on the cloud. Big data, Analytics, Web applications, Third party applications, collaboration and productivity tools to name few. Big bang approach and using same cloud offering to all blend of applications will be disastrous. The better option is to perform an application assessment for prioritizing the workloads by considering factors such as complexity, early to market and business outcomes. Once your application migration portfolio is in place, then follow schedule, ensure security and compliance, use automation and cloud operational governance. Remember to get rid of exiting on-premise system as the earliest for improving ROI.

\section{References \\ Journals}

[1] IBM (n.d). Cloud Migration. https://www.ibm.com/

[2] Microsoft (n.d). The stages of your migration journey. https://azure.microsoft.com/ 
[3] Orban, Stephen (2016, November 01). 6 Strategies for Migrating Applications to the Cloud. https://aws.amazon.com/

[4] Vadalia, Tejas (2020, March 02), The ROI of moving to the cloud. https://www.capgemini.com/

[5] The Open Group (n.d). Cloud Computing for Business - Building ROI from Cloud Computing. http://www.opengroup.org/

[6] Siddiqui, Yahya (2018, October 25). Take your Overall Cloud ROI to the Next Stage with Azure Technology Training. https://www.quickstart.com/

\section{Book}

[7] Wang, Lizhe. Ranjan, Rajeev. Chen, Jiniun. Benatallah, Benatallah(2017). Cloud Computing. CRC Press 